\title{
INTERPOLATION OF OPERATORS FOR $\Lambda$ SPACES
}

\author{
BY ROBERT SHARPLEY
}

Communicated by Alberto Calderón, September 22, 1973

Lorentz and Shimogaki [2] have characterized those pairs of Lorentz $\Lambda$ spaces which satisfy the interpolation property with respect to two other pairs of $\Lambda$ spaces. Their proof is long and technical and does not easily admit to generalization. In this paper we present a short proof of this result whose spirit may be traced to Lemma 4.3 of [4] or perhaps more accurately to the theorem of Marcinkiewicz [5, p. 112]. The proof involves only elementary properties of these spaces and does allow for generalization to interpolation for $n$ pairs and for $M$ spaces, but these topics will be reported on elsewhere.

The Banach space $\Lambda_{\phi}[1$, p. 65] is the space of all Lebesgue measurable functions $f$ on the interval $(0, l)$ for which the norm

$$
\|f\|_{\phi}=\int_{0}^{l} f^{*}(s) \phi(s) d s
$$

is finite, where $\phi$ is an integrable, positive, decreasing function on $(0, l)$ and $f^{*}$ (the decreasing rearrangement of $|f|$ ) is the almost-everywhere unique, positive, decreasing function which is equimeasurable with $|f|$.

A pair of spaces $\left(\Lambda_{\phi}, \Lambda_{\psi}\right)$ is called an interpolation pair for the two pairs $\left(\Lambda_{\phi_{1}}, \Lambda_{\psi_{1}}\right)$ and $\left(\Lambda_{\phi_{2}}, \Lambda_{\psi_{2}}\right)$ if each linear operator which is bounded from $\Lambda_{\phi_{i}}$ to $\Lambda_{\psi_{i}}$ (both $i=1,2$ ) has a unique extension to a bounded operator from $\Lambda_{\phi}$ to $\Lambda_{\psi}$.

THEOREM (LORENTZ-SHIMOGAKI). A necessary and sufficient condition that $\left(\Lambda_{\phi}, \Lambda_{\psi}\right)$ be an interpolation pair for $\left(\Lambda_{\phi_{1}}, \Lambda_{\psi_{1}}\right)$ and $\left(\Lambda_{\phi_{2}}, \Lambda_{\psi_{2}}\right)$ is that there exist a constant $A$ independent of $s$ and $t$ so that

$$
\Psi(t) / \Phi(s) \leqq A \max _{i=1,2}\left(\Psi_{i}(t) / \Phi_{i}(s)\right)
$$

holds, where $\Phi(s)=\int_{0}^{s} \phi(r) d r, \cdots, \psi_{2}(t)=\int_{0}^{t} \Psi_{2}^{s}(r) d r$.

Proof. We only sketch the proof of the necessity since it is standard.

AMS (MOS) subject classifications (1970). Primary 46E30, 46E35, 47A30.

Key words and phrases. Lorentz $\Lambda_{\phi}$ space, decreasing rearrangement, interpolation of operators. 
Suppose there are numbers $s_{n}$ and $t_{n}$ in $(0, l)$ such that $\Psi\left(t_{n}\right) / \Phi\left(s_{n}\right)>$ $n^{3} \max _{i=1,2}\left(\Psi_{i}\left(t_{n}\right) / \Phi_{i}\left(s_{n}\right)\right)$. Define the positive operator

$$
T_{n} f(t)=\left(C_{n} \int_{0}^{s_{n}} f(s) d s / s_{n}\right) \chi_{\left(0, t_{n}\right)}(t),
$$

where $C_{n}=\min _{i=1,2}\left(\Phi_{i}\left(s_{n}\right) / \Psi_{i}\left(t_{n}\right)\right)$.

For each $f$ in $\Lambda_{\phi_{i}}, T_{n} f$ belongs to $\Lambda_{\psi_{i}}$ and $T_{n}$ has operator norm less than or equal to 1 , but as an operator from $\Lambda_{\phi}$ to $\Lambda_{\psi}, T_{n}$ has operator norm larger than $n^{3}$. Hence the operator $T=\sum_{1}^{\infty} T_{n} / n^{2}$ is a bounded operator from $\Lambda_{\phi_{i}}$ to $\Lambda_{\psi_{i}}(i=1,2)$, but $T$ is not a bounded operator from $\Lambda_{\phi}$ to $\Lambda_{\psi}$.

To show that condition $(*)$ is sufficient, we prove that

$$
\|T f\|_{\psi} \leqq 2 A M\|f\|_{\phi}
$$

where $M$ is the maximum of the operator norms of $T$ acting from $\Lambda_{\phi}$ to $\Lambda_{\psi_{i}}(i=1,2)$. We can assume that $f$ is an arbitrary simple function with finite support since these functions are dense in $\Lambda_{\phi}$. We can also require $f$ to be positive since $\|f\|_{\phi}=\||f|\|_{\phi}$. Each function of this type can be written as $f=\sum_{1}^{n} \alpha_{i} \chi_{E_{i}}$ where the $\alpha_{i}{ }^{\prime}$ s are positive and $E_{n} \subset \cdots \subset E_{1}$. Hence $f^{*}=\sum_{1}^{n} \alpha_{i} \chi_{\left(0, a_{i}\right)}$ where $a_{i}=m E_{i}$. But then

$$
\left\|T \chi_{E}\right\|_{\psi} \leqq 2 A M \Phi(m E), \quad \text { all measurable } E \subset(0, l)
$$

is equivalent to relation (1), since

$$
\|T f\|_{\psi} \leqq \sum_{1}^{n} \alpha_{i}\left\|T \chi_{E}\right\|_{\psi} \leqq 2 A M \sum_{1}^{n} \alpha_{i} \Phi\left(a_{i}\right)=2 A M\|f\|_{\phi} .
$$

Hence, if we let $g=\left(T \chi_{E}\right)^{*}$, the proof is reduced to the following

Lemma. Suppose condition (*) holds and $g$ is a positive decreasing function that satisfies

$$
\|g\|_{\psi_{i}} \leqq M \Phi_{i}(a) \quad(i=1,2)
$$

then

$$
\|g\|_{\psi} \leqq 2 A M \Phi(a) .
$$

Proof. Firsı assume $g$ is a step function. with finite support, i.e., $g=\sum_{1}^{m} \beta_{j} \chi_{\left(0, t_{j}\right)}$. Set $J=\left\{j \mid \max _{i=1,2}\left(\Psi_{i}\left(t_{j}\right) / \Phi_{i}(a)\right)=\Psi_{1}\left(t_{j}\right) / \Phi_{1}(a)\right\}$ and then let $g_{1}=\sum_{j \in J} \beta_{j} \chi_{\left(0, t_{j}\right)}$ and $g_{2}=g-g_{1}$. Notice that both functions are positive, decreasing, step functions and

$$
\left\|g_{i}\right\| \leqq\|g\|
$$


in any $\Lambda$ space. Now using condition (*), relations (5) and (3), we have

Similarly

$$
\begin{aligned}
\left\|g_{1}\right\|_{\psi} / \Phi(a) & =\sum_{j \in J} \beta_{j} \Psi\left(t_{j}\right) / \Phi(a) \\
& \leqq A \sum_{j \in J} \beta_{j} \max _{i=1,2}\left(\Psi_{i}\left(t_{j}\right) / \Phi_{i}(a)\right) \\
& =A \sum_{j \in J} \beta_{j} \Psi_{1}\left(t_{j}\right) / \Phi_{1}(a)=A\left\|g_{1}\right\|_{\psi_{1}} / \Phi_{1}(a) \\
& \leqq A\|g\|_{\psi_{1}} / \Phi_{1}(a) \leqq A M .
\end{aligned}
$$

$$
\left\|g_{2}\right\|_{\psi} / \Phi(a) \leqq A\left\|g_{2}\right\|_{\psi_{2}} / \Phi_{2}(a) \leqq A M .
$$

Hence, we obtain relation (4) for positive, decreasing, step functions.

Now suppose $g$ is an arbitrary positive decreasing function and let $\left\{g_{n}\right\}$ be a monotone increasing sequence of positive decreasing step functions converging pointwise to $g$. By (3) and (5)

so

$$
\left\|g_{n}\right\|_{\psi_{i}} \leqq M \Phi_{i}(a) \quad(i=1,2)
$$

$$
\left\|g_{n}\right\|_{\psi} \leqq 2 A M \Phi(a)
$$

Applying the monotone convergence theorem to $\left\{g_{n} \psi\right\}$, we obtain relation (4).

The author wishes to thank Professor S. D. Riemenschneider for many helpful conversations regarding this work.

\section{REFERENCES}

1. G. G. Lorentz, Bernstein polynomials, Mathematical Expositions, no. 8, Univ. of Toronto Press, Toronto, 1953. MR 15, 217.

2. G. G. Lorentz and T. Shimogaki, Interpolation theorems for operators in function spaces, J. Functional Analysis 2 (1968), 31-51. MR 41 \#2424.

3. - Interpolation theorems for spaces $\Lambda$, Abstract Spaces and Approximation (Proc. Conf., Oberwolfach, 1968), Birkhäuser, Basel, 1969, pp. 94-98. MR 41 \#2423.

4. R. C. Sharpley, Spaces $\Lambda_{\alpha}(X)$ and interpolation, J. Functional Analysis 11 (1972), 479-513.

5. A. Zygmund, Trigonometric series. Vol. II, 2nd ed. reprinted with corrections and some additions, Cambridge Univ. Press, New York, 1968. MR 38 \#4882.

Department of Mathematics, Oakland University, Rochester, Michigan 48063 